\title{
Microlensing: A Tool to Probe Distant Binary Populations
}

\author{
Rosanne Di Stefano \\ Department of Physics and Astronomy, Tufts University, Medford, MA \\ 02155 \\ Harvard-Smithsonian Center for Astrophysics, Cambridge, MA 02138
}

\begin{abstract}
Programs designed to observe gravitational microlensing are poised to provide a great deal of information about binary populations far from Earth, including those in the Galactic Bulge, in the Magellanic Clouds, in M31, and perhaps in other external galaxies. Because many millions of stars are monitored, microlensing observations allow us to study binaries in three ways: (1) when they are "involved" in a microlensing event (as either a lens or lensed source), (2) when variability due to binarity produces significant variations in the light curve, and (3) when light from a more distant star is attenuated or refracted by matter associated with the binary system (e.g., in a disk).

Microlensing observations will discover large numbers of binaries and planetary systems in a variety of galactic environments. Thus, comparative statistical studies of binary properties (distributions of mass ratios and orbital separations) are possible.

An intriguing sign that we have already begun to learn about binaries through microlensing observations comes from work indicating that all of the lenses detected to date may in fact be binaries. For observations along the direction of the Magellanic Clouds this would imply that, if the lenses are primarily located in the Halo, then MACHOs tend to be binaries. If, on the other hand, most of the lenses are located in the Magellanic Clouds, microlensing observations are giving us a unique way to explore a distant stellar population of binaries.
\end{abstract}

\section{Introduction}

We began this meeting by considering the history of the field. It is now fitting that we end with a look toward the future. Although microlensing programs were not designed to study binaries, they are nevertheless poised to provide important and unique insights into the characteristics of binary populations.

Observations designed to detect evidence of gravitational microlensing typically involve nightly monitoring of the flux received from tens of millions of stars. Such observations can teach us about binaries in three ways.

(1) When a binary system serves as a lens or as a source of light that is lensed, it may be possible to constrain the values of the binary mass, mass ratio, projected 
orbital separation of the binary, and transverse velocity of the lens with respect to the line of sight to the source star.

(2) The light curve associated with the binary itself may be time variable, due to, e.g., eclipses or accretion processes.

(3) Light from a distant star may experience electromagnetic interactions with matter associated with the binary, e.g., in a disk (Di Stefano \& Keeton 2000). Such interactions can cause the light to be attenuated or even to be refracted.

In this talk I will discuss what the microlensing observations have contributed thus far, but will focus on providing an overview of their broad capabilities to contribute to our knowledge of binary populations. As I hope to demonstrate, the possibilities are wonderfully diverse. No single "bottom line" can therefore summarize what we may hope to learn about binaries from the microlensing observations. There are, however, some simple expectations. These are derived from the fact that observations designed to discover evidence of microlensing probe such vast volumes of our own and other galaxies that they will provide an unprecedented statistical view of binary populations and of planetary systems as a function of galactic environment. This will provide insight into the circumstances and frequency of binary formation.

\section{Gravitational Microlensing}

Gravitational lensing is simply the phenomenon in which the path of light is affected by gravitational interactions. If a point source of light, a point lens of mass $M$, and the observer are perfectly aligned, then the image of the source is a ring of angular dimension, $\theta_{E}$, the Einstein angle. Let $D_{L}$ represent the distance from the observer to the lens, and $x$ be the ratio of $D_{L}$ to $D_{S}$, the distance to the lensed source.

$$
\theta_{E}=\sqrt{\frac{4 G M(1-x)}{c^{2} D_{L}}}=0.003 " \sqrt{(1-x)\left(\frac{M}{M_{\odot}}\right)\left(\frac{k p c}{D_{L}}\right)}
$$

The associated Einstein radius as projected onto the lens plane is

$$
R_{E}=\sqrt{\frac{4 G M D_{S} x(1-x)}{c^{2}}}=9.0 A U \sqrt{x(1-x)\left(\frac{M}{M_{\odot}}\right)\left(\frac{D_{S}}{10 k p c}\right)}
$$

Alignment that is not perfect leads to multiple distorted images of the source. If the lens is a point mass, there will be 2 images, with characteristic angular separations comparable to $\theta_{E}$. For stellar-mass lenses located at distances on the order of a $\mathrm{kpc}$ from the observer, $\theta_{E}$ is typically too small to allow ground-based optical telescopes to resolve the images.

Evidence of lensing can nevertheless be obtained by monitoring the luminosity of the lensed star. Magnification of the images leads to an enhancement in the received light. Let $u$ be the projected separation between the lens and source, measured in units of $R_{E}$. The magnification $A(u)$ is

$$
A(u)=\frac{u^{2}+2}{u \sqrt{u^{2}+4}} .
$$




\section{Point Lens}

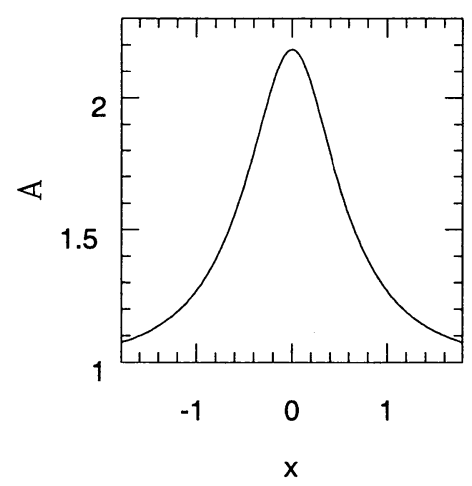

Figure 1. The magnification due to a point mass. In the case shown, the distance of closest approach between the projected position of the source and the lens is $0.5 R_{E}$. Note that point-lens events are highly degenerate, as only a single measurement, the time duration of the event, can be used to constrain the combination of $M_{l e n s}, D_{S}, D_{L}$, and $v_{t}$.

The Einstein time is the time taken for the source to traverse a distance $2 R_{E}$; significant changes in magnification can take place on much shorter time scales.

$$
t_{E}=17.4 \text { days }\left(\frac{R_{E}}{A U}\right)\left(\frac{100 \mathrm{~km} / \mathrm{s}}{v_{t}}\right) .
$$

The optical depth is defined to be the probability that a microlensing event is occurring: it is roughly equal to the fraction of the sky enclosed by Einstein rings. It is typically a very small number: e.g., the optical depth due to stellar lenses located along the direction to Baade's window is on the order of $10^{-6}$.

\section{Monitoring Many Stars}

Because the probability of lensing is so small, it is impractical to wait for any given star to be lensed. Programs to observe microlensing events therefore concentrate on dense fields, to monitor the light received from tens of millions of stars. The Magellanic Clouds are monitored because they are located behind a significant portion of the Galactic Halo, and because the optical depth due to known stellar populations was initially thought to be small enough (roughly an order of magnitude smaller than for Baade's window), that any signal from MACHOs would be readily detected. The Galactic Bulge, as seen through Baade's window, is also an important target in that it serves as a "control" field; lensing along this direction is expected to be mostly due to stars.

The microlensing teams monitor multiple fields each night, having created a template of point sources for each field. During the first years of observations, variable stars among the template stars were identified. The teams then concentrated on looking for changes in flux from any of the template stars that had not 


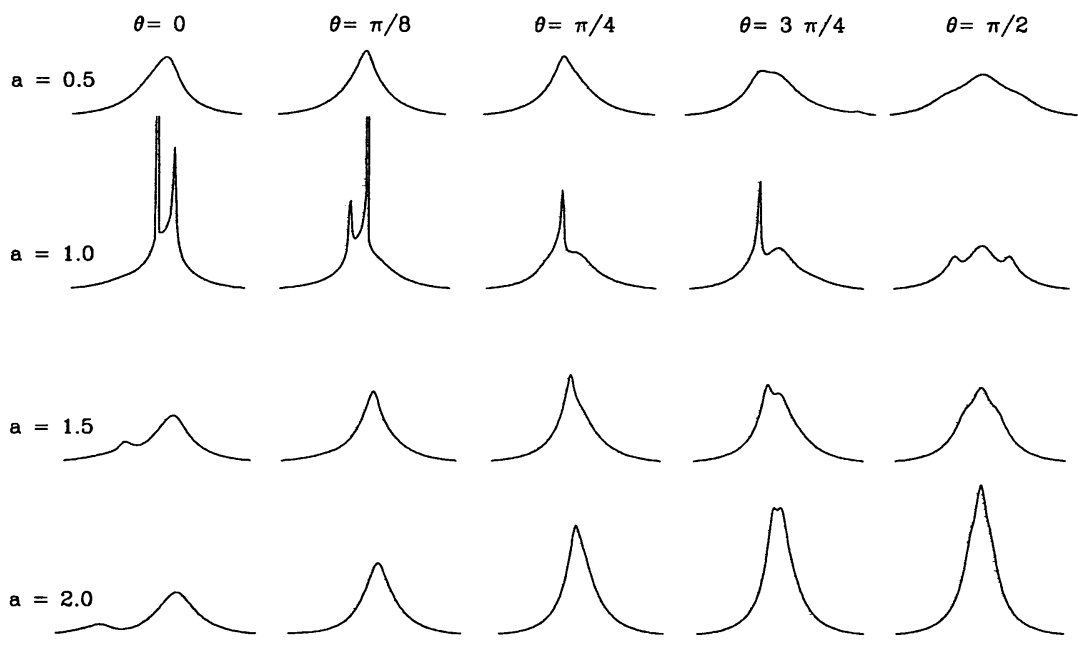

Figure 2. The magnification due to binary lenses. In all cases the mass ratio is 0.5 and the distance of closest approach between the projected position of the source and the lens is $0.5 R_{E}$. Note the diversity of shapes: lens binarity introduces structure that can help break the degeneracy inherent in point-lens fits. Only 2 of these light curves ( $\left.a=1.0 R_{E}, \theta=0, \pi / 8\right)$ exhibit caustic crossings.

previously been observed to deviate from the measured baseline. Most of the more-than-400 events discovered to date have been discovered using the criterion of an acceptable point-lens fit to identify promising candidates for microlensing events.

\section{Binary Lenses}

When the lenses are binaries, the situation is quite different. There are either 3 or 5 images, and the isomagnification curves are no longer axisymmetric. Nevertheless, for projected binary separations less than roughly $0.1 R_{E}$ and greater than approximately $3 R_{E}$, deviations from the standard Paczyński light curve tend to be small, and only a small fraction of all light curves are significantly perturbed.

For binaries with $0.1 R_{E}<a<3 R_{E}$, however, a large fraction of all light curves are recognizably different from the point-lens form, exhibiting a wide variety of shapes (see Figure 2). The most distinctive light curves are those exhibiting the wall-like structures associated with caustic crossings; caustic crossings occur when the track of the lensed star passes just behind the caustic curves that separate regions in which there are 3 images from regions in which there are 5 images. 


\subsection{Most of the Lenses May Be Binaries}

The relative numbers of caustic-crossing, more gently perturbed, and point-lenslike light curves depend on the linear dimensions of the caustic structures. For each lens geometry, as defined by the projected orbital separation of the binary components and their mass ratio, these relative numbers can be determined either through a pictorial examination of the caustic structures, or through a set of scattering experiments. The results are that, even for the large values of $q$ that maximize the probability of a caustic crossing, there is only 1 caustic-crossing event for every $4.5-5$ events. Furthermore, we must take into account the fact that most binary lenses have orbital separations closer than $0.1 R_{E}$ or larger than $3.0 R_{E}$. If the combination of such closer and wider binaries accounts for $2 / 3$ of all binary-lens events, then there should be 14 or more non-caustic-crossing events for every caustic-crossing event. We can observe significantly fewer noncaustic-crossing events per caustic-crossing light curve only if binary lenses with projected separations near $R_{E}$ are more numerous than other binaries, while there should be more non-caustic-crossing events if the distribution of mass ratios is peaked at values of $q$ smaller than $\sim 0.4$ (Di Stefano 2000).

When these simple consequences of the geometry of binary lenses are compared with observations, the result is striking. All of the microlensing monitoring teams that have been active for several years have observed caustic-crossing events, and the ratio of caustic-crossing to other events ranges from $1 / 20$ to $1 / 2$. The figure of $1 / 20$ comes from the data set with the largest number of events, the MACHO team's store of some 350 events along directions to the Galactic Bulge (Alcock et al. 1999). The OGLE I collaboration and the DUO team, with smaller numbers of events, have found the ratio of caustic-crossing to other events to be $\sim 1 / 12$ (Udalski et al. 1994; Alard, Mao, \& Guibert 1995). This is similar to the LMC results published by the MACHO team (Alcock et al. 1997a), although the latest results reduce the ratio to between 1/13 and 1/17 (Alcock et al. 2000). The ratio of $1 / 2$ comes from the SMC data set. Thus, in each data set, along every line of sight investigated so far, the ratio of the numbers of caustic-crossing events to other events is so high that it is consistent with a lens population composed entirely of binaries (DiStefano 1999, 2000). Indeed, the hypothesis that all of the lenses are binaries cannot presently be falsified. If the lenses are primarily located in the Galactic Halo, then we may be on the verge of establishing the intriguing result that MACHOs travel in pairs.

\subsection{The Magellanic-Cloud Events}

I have made the case that each caustic-crossing event can essentially carry with it $\sim 10$ or more other events, most of which are point-lens-like, and that these additional events may comprise the majority of the events discovered so far. Thus, if we could discover the location of the lenses responsible for the causticcrossing events, we would also know the location of most of the other lenses. In fact, we have been able to determine that the most likely lens location for each of the two caustic-crossing events along directions to the Magellanic Clouds is in the Clouds themselves (see, e.g., Alcock et al. 1997a, Afonso et al. 2000). This determination depends on being able to use information about the caustic crossing to constrain the value of the transverse velocity, and then comparing the value so derived with the probability distributions of transverse velocities 
expected for lenses in the Galactic Halo and in the Magellanic Clouds. The discovery and study of a relatively small number of additional caustic-crossing events would provide the simplest route to establish that the majority of the lenses are in the Magellanic Clouds, should that prove to be true, or that a significant fraction of the lenses are Halo objects (Di Stefano 1999, 2000). Thus, each caustic-crossing event subject to the sort of intensive study accorded 98SMC-1 (Afonso et al. 2000 and references therein) has the potential not only to break the degeneracy in the physical parameters associated with that event, but also to determine the location of a large number of other lenses and to thereby help determine the MACHO Halo fraction.

\subsection{A Puzzle and a Challenge}

If most lenses are binaries, then there are many events with characteristics distinguishing them from point-lens events, but which do not exhibit caustic crossings. In fact, the calculations summarized above indicate that there should be roughly $2-4$ times as many such events as there are caustic-crossing events. Yet, few such events have been observed. Also missing from the mix of observed events are binary-source events. This clearly indicates that a fairly significant fraction of events have either been missed or misidentified. Work to understand what this means for the microlensing observations is underway.

\section{Ongoing and Future Observations}

The MACHO team has now ceased observations, while other teams continue with programs that, like EROS II and OGLE II, have been and are continuing to be upgraded. One of the most important improvements beginning to be implemented is the use of image differencing techniques to identify variability. This means that we can now detect modest variability of stars not bright enough to be visible on a pre-measured template. Image differencing techniques are now being applied (although not necessarily in real time) to all of the monitored fields, including M31. In fact the monitoring of M31 is one of the very interesting new developments in the microlensing observations. Even in fields already monitored, however, image differencing will increase the rate of observed events by a factor of $2-3$. New camera systems can lead to additional increases. Within the next few years we may be detecting hundreds of events per year in Baade's window and tens of events per year toward the Magellanic Clouds. Such numbers, particularly toward the Bulge, will allow us to develop ensembles of events large enough to provide fodder for meaningful statistical analyses, and will also discover relatively rare events.

\section{Planets}

Before the microlensing observations began, it was recognized that binaries could be detected, with the special case of small-mass-ratio binaries corresponding to planetary systems (Mao \& Paczyński 1991). Gould \& Loeb (1992) focused on the planetary case, in part spurred on by the following coincidence. If a system identical to our own solar system, but seen face-on and located in the 
Galactic disk, were to serve as a lens for a Bulge star, Jupiter would be located approximately $1 R_{E}$ from the sun. They estimated that $\sim 15 \%$ of all source tracks giving rise to a lensing event would contain evidence of Jupiter's presence. The evidence would be in the form of a sharp but short-lived perturbation from the Paczński light curve due to lensing by the star.

Once microlensing events had been discovered, more attention was focused on discovering planets via microlensing. In fact, the microlensing programs were augmented to achieve the goal of planet detection. The monitoring teams began to call alerts when they detected a deviation from baseline they were reasonably sure was due to microlensing. These alerts would trigger more frequent observations with sensitive photometry, carried on at enough locations in the Southern hemisphere to achieve 24-hour coverage, at least when the weather cooperated. (See, e.g., Albrow et al. 1998.) It was hoped that this aggressive approach would enable microlensing to become the first method to discover extra-solar planets. Of course we now know that the results have not lived up to these early hopes. With $\sim 30$ extra-solar planets discovered, there has not been a single light curve observed with characteristics that can be uniquely ascribed to the presence of a planet.

It is natural to ask whether this means that microlensing is a technique not well-suited to the discovery of planets. For individual well-monitored light curves that show no evidence of a planet, we can compute the level of confidence at which we can rule out the presence of planets with various mass ratios and projected separation from the central star. Less massive planets are difficult to detect, because of the effects of both finite-source-size and blending. Planets outside of a thin annulus $\left(0.6-1.6 R_{E}\right)$ are essentially impossible to detect using the observing strategy employed to date. With only a relative handful of light curves so far subjected to observations detailed enough to detect planets in this thin annulus, it is clear that we will need to study more light curves in order to either discover planets in the annulus or to place meaningful limits on the population of planets.

Perhaps even more important, we must extend the search to include planets in wider orbits (Di Stefano \& Scalzo 1999a,b). Detecting such planets requires good photometry and sensitivity to (a) events of short duration, and/or to (b) events which are perturbed from the Paczyński form on the rise from or fall back to baseline. No systematic searches for planets in wider orbits have yet been implemented. Nevertheless, improvements in the observations, such as those described in $\S 4$, could well lead even ongoing programs to the discovery of $\sim 10$ planets per year in Baade's window, and smaller numbers in other monitored fields. It seems likely that, during the next decade, microlensing observations will either discover planets in distant locations and begin to establish the statistics of mass ratios and orbital separations, or will place meaningful limits establishing the differences between the solar neighbourhood and other places, such as the Bulge, with regard to the frequency and characteristics of planetary systems.

\section{Variability}

There is a romance to the study of variable stars, with individual observers adopting certain variables as their own, and returning to them again and again. 
The microlensing surveys represent a change in philosophy and practice, inspired by the ability to obtain and store vast quantities of data. Fields containing millions of stars are monitored night after night. Given the prevalence of stellar binarity and the large fraction of binaries that are variable, the monitored fields are certain to include many thousands of variable binaries.

The OGLE team has identified 1459 eclipsing binary stars in the central 2.4 square degree area of the Small Magellanic Cloud (Udalski et al. 1998), while the MACHO team has reported on 611 eclipsing binary stars in the Large Magellanic Cloud (Alcock et al. 1997). The OGLE team has instituted regular studies of both open clusters and globular clusters. In the young open cluster NGC 5999, 30 eclipsing binaries have been found (Pietrzynski et al. 1998), while in NGC 5381, 13 eclipsing variables were found (Pietrzynski et al. 1997); among the variables discovered are W UMa and Algol-like systems. The OGLE team also identified 12 eclipsing binaries in the globular cluster 47 Tuc (Kaluzny et al. 1998), while surveys of 3 fields covering the central part of the globular cluster $\omega$ Cen identified 10 SX Phe stars and 19 eclipsing binaries, most of which were not previously known (Kaluzny et al. 1997a, b). In addition, further study of 3 detached binaries discovered in their sample can provide direct information about properties of the turnoff stars in $\omega$ Cen. It is interesting to note that the strategies used by the microlensing teams have been so successful that they are presently being applied specifically to the study of cluster variables, independent of any lensing applications. (See, e.g., Kaluzny 1999, Olech 1999.) Monitoring by the microlensing teams has also helped us to characterize the optical variability of a new class of sources, luminous binary supersoft sources (LBSSSs; Greiner et al. 1999). There will certainly be many other such results that are difficult to anticipate.

\section{Disks}

Gravitational microlensing is just one example of an interaction between light from a distant star and intervening matter. Electromagnetic interactions can also lead to time variability that can provide clues about the existence and characteristics of the intervening matter, and even about characteristics of the source star. Draine (1998) has considered refraction effects that might be associated with spherical clouds.

An example of particular relevance to star formation is attenuation, or even refraction, due to disks. Di Stefano \& Keeton (2000) have considered microlensing, refraction, and attenuation due to disks. Although the signature from just one of these effects can contain a good deal of structure, and it may even be necessary to consider more than one effect in some systems, typical time durations for signals associated with refraction and/or attenuation can be estimated in a straightforward manner. Consider a disk with axes, as projected onto the plane of the sky, of width $w$ and length $l$. With $w<l$, the shortest disturbance lasts for time $w / v_{t}$. If $w$ is on the order of an $\mathrm{AU}$, the time durations of events will be comparable to those of microlensing events due to lensing by stars. 


\section{The Future}

There are powerful reasons for collaborative efforts between the community of scientists interested in binaries and the community of researchers studying microlensing. The motivation of the binary community is clear, since the microlensing data sets contain much information about binaries - whether these binaries serve as lenses, lensed sources, exhibit intrinsic variability, or interact electromagnetically with light on its way to us from a more distant source. On the other hand, the microlensing community is beginning to realize that being alert to signals from binaries may be important for the derivation of meaningful results about MACHOs. This is because (1) individual binary-lens events can break the degeneracy inherent in the so-called Paczyński light curve, (2) simple properties of ensembles of binary-lens events can point to problems, e.g., alerting us to events that have either been missed or misidentified, (3) the combination of unrecognized lensing by binaries, combined with the effects of blending, could lead to errors in the estimate of the optical depth. In fact, there is an excess in the measured values relative to the value predicted based on stellar populations even along the direction to the Bulge, the so-called "control" fields. This discrepancy is almost certainly due to something intrinsic to the analysis of ordinary stellar populations rather than dark matter; it is therefore clearly important to systematically include the effects of binarity in the analyses.

Given these powerful motivations from each community, it seems clear that the methods developed to study microlensing will become powerful tools for the study of binaries. There should be much more to report in our next meeting.

Acknowledgments. This work was supported by NSF under grant AST9619516.

\section{References}

Afonso, C., et al. 2000, ApJ, 532, 340

Alard, C., Mao, S., \& Guibert, J. 1995, A\&A, 300L, 17

Albrow, M., et al. 1998 ApJ, 509, 687

Alcock, C., et al. 2000, astro-ph/0001272

Alcock, C., et al. 1999, astro-ph/9907369

Alcock, C., et al. 1997a, ApJ, 486, 697

Alcock, C., et al. 1997b, ApJ, 479, 119

Di Stefano, R. 2000, ApJ, 541, 587

Di Stefano, R. 1999, astro-ph/9901035

Di Stefano, R., \& Scalzo, R. A. 1999, ApJ, 512, 564

Di Stefano, R., \& Scalzo, R. A. 1999, ApJ, 512, 579

Di Stefano, R., \& Keeton, C. R. 2000, in prep.

Draine, B. T. 1998, ApJ, 509, 41

Gould, A., \& Loeb, A. 1992, ApJ, 396, 104

Greiner, J., Tovmassian, G. H., Di Stefano, R., et al. 1999, A\&A 343, 183

Kaluzny, J, et al. 1998, A\&AS, 128, 19 
Kaluzny, J, et al. 1997, A\&AS, 125, 343

Kaluzny, J, et al. 1997, A\&AS, 122, 471

Kaluzny, J., Thompson, I., Krzeminski, W., Pych, W. 1999, A\&A, 350, 469

Mao, S., \& Paczyński, B. 1991, ApJ, 374, L37

Olech, A. et al. 1999, MNRAS, 310, $759 \mathrm{O}$

Pietrzynski, G., Kubiak, M., Udalski, A., Szymanski, M. 1997, AcA, 47, 437

Pietrzynski, G., Kubiak, M., Udalski, A., Szymanski, M. 1998, AcA, 48, 489

Udalski, A. et al. 1994, ApJ, 436, L103.

Udalski, A. et al. 1998, AcA, 48, 563 